\title{
Integral equation formulations in 2D inhomogeneous magnetoelectroelastic media
}

\author{
C. Y. Dong \\ Department of Mechanics, School of Aerospace Engineering, \\ Beijing Institute of Technology, China
}

\begin{abstract}
Integral equation formulations for $2 \mathrm{D}$ inhomogeneous finite/infinite anisotropic magnetoelectroelastic media are presented. The present formulations only contain the fundamental solutions from the matrix which is taken as a homogeneous anisotropic magnetoelectroelastic medium. Functionally graded linear magnetoelectroelastic inclusions can be considered in which the corresponding fundamental solutions are not needed. In numerical implementation, inclusions are discretized into a series of quadratic quadrilateral or triangular elements, and cracks are meshed into a series of quadratic discontinuous boundary elements. For finite domain, the domain boundaries are discretized into a series of quadratic boundary elements. Finally, the present integral equation method can be used to investigate the interaction between cracks and inclusions in 2D anisotropic magnetoelectroelastic media and to carry out the analysis of effective properties of magnetoelectroelastic media.
\end{abstract}

Keywords: magnetoelectroelastic media, integral equation formulations, inhomogeneities, cracks.

\section{Introduction}

Magnetoelectroelastic composite materials have been receiving more attention in modern smart structure applications [1]. The study of inhomogeneous magnetoelectroelastic mechanics behavior has important theoretical and application values in the fields of electrics, microwave, supersonics, laser, infrared and so on [2].

Extensive investigations of the properties of magnetoelectroelastic composite materials have been carried out by many researchers. Based on the inclusion 
formulation, Huang and Kuo [3] presented a unified method to determine the magnetic, electric, and elastic fields in piezoelectric/piezomagnetic composite materials with the ellipsoidal inclusions. Huang et al. [4] obtained the magnetoelectroelastic Eshelby tensors for piezomagnetic/piezoelectric composite matrix containing an ellipsoidal inclusion. $\mathrm{Li}$ [5] studied the average magnetoelectroelastic field in multi-inclusions or inhomogeneities embedded in an infinite matrix and presented a numerical method to evaluate the magnetoelectroelastic Eshelby's tensors for the general material symmetry and ellipsoidal inclusion shape. Liu et al. [6] obtained Green's functions for anisotropic magnetoelectroelastic solids containing an elliptical cavity or a crack. Pan and his co-workers presented three dimensional Green's functions in anisotropic magnetoelectroelastic bimaterials [7] and exact solution for 2D polygonal inclusion problem in anisotropic magnetoelectroelastic full-, half-, and bimaterial-planes [8]. Hou and Leung [9] obtained the exact closed-solutions of the coupled field of a spheroidal magnetoelectroelastic inclusion embedded in an infinite magnetoelectroelastic matrix subjected to remote spatially homogeneous mechanical and electromagnetic loadings. Dinzart and Sabar [10] proposed a micromechanical model for the estimate of the magnetoelectroelastic behavior of the magnetic-piezoelectric composites with coated reinforcements. Shen and Hung [11] adopted complex variable and Faber series method to carry out magnetoelectroelastic analysis of an arbitrary shape inclusion with eigenfields embedded in an infinite domain subject to remote loadings. For more realistic cases with irregular geometric shapes and complex loadings, numerical methods should be used in the studies of various properties of magnetoelectroelastic inhomogeneities. Garcia-Sanchez et al. [1] obtained the solution of circular crack in magnetoelectroelastic media by means of the boundary element method. Dong et al. [12] carried out the analysis in 2D cracked magnetoelectroelastic media using the boundary element method. Pasternak [13] developed a two dimensional boundary element method to solve a magnetoelectroelastic medium with doubly periodic sets of cracks or thin inclusions. Based on the hypersingular formulation of the boundary element method, Rojas-Díaz et al. [14] presented a numerical approach to analyze multiple cracks with different crack face boundary conditions in 2D magnetoelectroelastic media.

In this paper, the integral equation formulations of 2-D inhomogeneous finite/infinite magnetoelectroelastic media are presented. The integral formulations only contain the extended discontinuous displacements (elastic displacement, electrical potential and magnetic potential) over the cracks and the extended fundamental solutions from the homogeneous magnetoelectroelastic matrix. The inclusions may be functionally graded or other complex inhomogeneous materials. The interaction between inclusions and cracks can be studied using the present formulations.

\section{Basic formulations}

For an inclusion occupying a domain $\Omega_{I}$ enclosed by boundary $\Gamma_{I}$ (i.e. the interface between the inclusion and matrix) embedded in a magnetoelectroelastic 
matrix, the stress $\sigma_{i j}$, the electric displacement $D_{i}$ and the magnetic induction $B_{i}$ are as follows [8]

$$
\sigma_{i J}=C_{i J K l} \gamma_{K l}
$$

with

$$
\begin{gathered}
\gamma_{I j}=\left\{\begin{array}{cc}
\gamma_{i j}, & (I=1,2,3) \\
-E_{j}, & (I=4) \\
-H_{j} & (I=5)
\end{array}\right. \\
\sigma_{i J}=\left\{\begin{array}{cc}
\sigma_{i j}, & (J=1,2,3) \\
D_{i}, & (J=4) \\
B_{i}, & (J=5)
\end{array}\right. \\
=\left\{\begin{array}{lc}
C_{i j k l}, & J, K=1,2,3 \\
e_{l i j} & J=1,2,3 ; K=4 \\
e_{i k l} & J=4 ; K=1,2,3 \\
q_{l i j} & J=1,2,3 ; K=5 \\
q_{i k l} & J=5 ; K=1,2,3 \\
-\lambda_{i l} & J=4, K=5 ; J=5, K=4 \\
-\varepsilon_{i l} & J=K=4 \\
-\mu_{i l} & J=K=5
\end{array}\right.
\end{gathered}
$$

where $\gamma_{i j}$ are the strain, $E_{j}$ are the electric field and $H_{j}$ are the magnetic field, respectively; $C_{i j k l}$ are the elastic moduli, $e_{l i j}$ are the piezoelectric constants, $q_{l i j}$ are the piezomagnetic constants, $\lambda_{i l}$ are the electromagnetic constants, $\varepsilon_{i l}$ are the dielectric permittivities, and $\mu_{i l}$ are the magnetic permeabilities, respectively. In eqn (1), and throughout this paper, the usual index notations are adopted with lower case subscripts ranging over (1-3) and capital case subscripts ranging over (1-5), respectively. Repeated indices imply summation.

Assumed that the extended elastic moduli of the magnetoelectroelastic matrix are expressed as $C_{i J K l}^{M}$ which are taken as the constants for any points in the matrix. Thus, the extended elastic moduli of the inhomogeneous magnetoelectroelastic inclusion can be written as $C_{i J K l}=C_{i J K l}^{M}+\Delta C_{i J K l}$ in which $\Delta C_{i J K l}$ are the difference between the extended elastic moduli from the inclusion and matrix. Therefore, eqn (1) can be rewritten as 


$$
\sigma_{i J}=\left(C_{i J K l}^{M}+\Delta C_{i J K l}\right) \gamma_{K l}
$$

Based on the principle of virtual work, one has

$$
\int_{\Omega_{I}} C_{i J K l} \gamma_{K l} \gamma_{J i}^{*} d \Omega=\int_{\Gamma_{I}} \sigma_{i J} n_{i} u_{J}^{*} d \Gamma
$$

where $u_{J}^{*}$ is the virtual displacement component; $\gamma_{J i}^{*}$ is the corresponding virtual strain.

Using $C_{i J K l}=C_{i J K l}^{M}+\Delta C_{i J K l}$, eqn (4) can be given as

$$
\int_{\Omega_{I}} C_{i J K l}^{M} \gamma_{K l} \gamma_{J i}^{*} d \Omega=\int_{\Gamma_{I}} \sigma_{i J} n_{i} u_{J}^{*} d \Gamma-\int_{\Omega_{I}} \Delta C_{i J K l} \gamma_{K l} \gamma_{J i}^{*} d \Omega
$$

Considering $\sigma_{l K}^{*} \gamma_{K l}=\sigma_{l K}^{*} u_{K, l}=\left(\sigma_{l K}^{*} u_{K}\right)_{, l}-\sigma_{l K, l}^{*} u_{M}$ in which the index notation following a comma signifies differentiation with respect to spatial coordinates, eqn (5) becomes

$$
\int_{\Gamma_{I}} \sigma_{l K}^{*} n_{l} u_{K} d \Omega-\int_{\Omega_{I}} \sigma_{l K, l}^{*} u_{K} d \Omega=\int_{\Gamma_{I}} \sigma_{i J} n_{i} u_{J}^{*} d \Gamma-\int_{\Omega_{I}} \Delta C_{i J K l} \gamma_{K l} \gamma_{J i}^{*} d \Omega(6)
$$

Following Brebbia and Dominguez [15], the fundamental solution can be obtained for an extended point load $\Delta(P)=0$ along the direction of the unit vector $e_{K}=0$ using the following equation

$$
\sigma_{l K, l}^{*}+\Delta(P) e_{K}=0
$$

The fundamental solutions can be written as

$$
\begin{aligned}
& u_{J}^{*}=U_{I J}^{*} e_{I} \\
& t_{J}^{*}=T_{I J}^{*} e_{I} \\
& \gamma_{J i}^{*}=U_{I J, i}^{*} e_{I}
\end{aligned}
$$

where $U_{I J}^{*}, T_{I J}^{*}$ are $J$ components of the extended displacements and tractions due to an extended unit point load in the $I$ direction. The expressions of the fundamental solutions $U_{I J}^{*}, T_{I J}^{*}$ and $U_{I J, i}^{*}$ can be found in the reference [1]. The second integral of the left hand side in eqn (6) for a particular direction $e_{K}$ of the extended unit load can be given as 


$$
\int_{\Omega_{I}} \sigma_{l K, l}^{*} u_{K} d \Omega=-\int_{\Omega_{I}} \Delta(P) u_{I} e_{I} d \Omega=u_{I}(P) e_{I}
$$

where $u_{I}(P)$ is the $I$-th component of the extended displacements at the source point $P$ with coordinates $\left(x_{1}(P), x_{2}(P)\right)$.

Substituting eqns (8) and (9) into eqn (6), one can obtain

$$
\begin{aligned}
u_{I}(P)= & \int_{\Gamma_{I}} U_{I J}^{*}(P, q) t_{J}(q) d \Gamma(q)-\int_{\Gamma_{I}} T_{I J}^{*}(P, q) u_{J}(q) d \Gamma(q)- \\
& \int_{\Omega_{I}} \Delta C_{i J K l}(Q) \gamma_{K l}(Q) U_{I J, i}^{*}(P, Q) d \Omega(Q)
\end{aligned}
$$

where $q$ with coordinates $\left(x_{1}(q), x_{2}(q)\right)$ and $Q$ with coordinates $\left(x_{1}(Q), x_{2}(Q)\right)$ denote the field points over the interface $\Gamma_{I}$ and domain $\Omega_{I}$, respectively.

Considering that the source point $P$ is in the matrix, the above equation becomes

$$
\begin{aligned}
0= & \int_{\Gamma_{I}} U_{I J}^{*}(P, q) t_{J}(q) d \Gamma(q)-\int_{\Gamma_{I}} T_{I J}^{*}(P, q) u_{J}(q) d \Gamma(q)- \\
& \int_{\Omega_{I}} \Delta C_{i J K l}(Q) \gamma_{K l}(Q) U_{I J, i}^{*}(P, q) d \Omega(Q)
\end{aligned}
$$

For the matrix, one has the following equation

$$
\begin{aligned}
u_{I}(P)= & \int_{\Gamma} U_{I J}^{*}(P, q) t_{J}(q) d \Gamma(q)-\int_{\Gamma} T_{I J}^{*}(P, q) u_{J}(q) d \Gamma(q)+ \\
& \int_{\Gamma_{I}} U_{I J}^{*}(P, q) t_{J}(q) d \Gamma(q)-\int_{\Gamma_{I}} T_{I J}^{*}(P, q) u_{J}(q) d \Gamma(q)
\end{aligned}
$$

where $\Gamma$ is the outer boundary of the matrix.

Along the interface $\Gamma_{I}$, the extended displacements from the inclusion side and matrix side must be equal. Besides, the extended tractions along the interface remain the equilibrium. Thus, one can obtain by adding eqns (11) and (12)

$$
\begin{gathered}
u_{I}(P)=\int_{\Gamma} U_{I J}^{*}(P, q) t_{J}(q) d \Gamma(q)-\int_{\Gamma} T_{I J}^{*}(P, q) u_{J}(q) d \Gamma(q)- \\
\int_{\Omega_{I}} \Delta C_{i J K l}(Q) \gamma_{K l}(Q) U_{I J, i}^{*}(P, Q) d \Omega(Q)
\end{gathered}
$$

The above equation contains domain integrals over the whole inclusion which need to be discretized. If the fundamental solutions for the inclusions are 
available, the subdomain boundary element method can be used to solve these magnetoelectroelastic inhomogeneities.

When $P$ approaches the boundary $\Gamma$, eqn (13) becomes

$$
\begin{gathered}
C_{I J}(P) u_{J}(P)= \\
\int_{\Gamma} U_{I J}^{*}(P, q) t_{J}(q) d \Gamma(q)-\int_{\Gamma} T_{I J}^{*}(P, q) u_{J}(q) d \Gamma(q)- \\
\int_{\Omega_{I}} \Delta C_{i J K l}(Q) \gamma_{K l}(Q) U_{I J, i}^{*}(P, Q) d \Omega(Q)
\end{gathered}
$$

where $C_{I J}$ are the free terms depending upon the local geometry at the source point $P$.

To find the solution of the above equation, the integral representation for the extended strain tensor is also needed. Assume that the source point $P$ lies inside the inclusion and let $\Omega_{e}$ enclosed by the boundary $\Gamma_{e}$ denote a portion of $\Omega_{I}$ containing $P$ as an interior point. Since

$$
\int_{\Omega_{e}} U_{I J, i}^{*}(P, Q) d \Omega(Q)=\int_{\Gamma_{e}} U_{I J}^{*}(P, q) n_{i}(q) d \Gamma(q)
$$

So we can obtain the following equation

$$
\int_{\Omega_{e}} \Delta C_{i J K l}(P) \gamma_{K l}(P) U_{I J, i}^{*}(P, Q) d \Omega(Q)=\int_{\Gamma_{e}} \Delta C_{i J K l}(P) \gamma_{K l}(P) U_{I J}^{*}(P, q) n_{i}(q) d \Gamma(q)
$$

Adding the above equation from eqn (13), one can obtain

$$
\begin{gathered}
u_{I}(P)=\int_{\Gamma} U_{I J}^{*}(P, q) t_{J}(q) d \Gamma(q)-\int_{\Gamma} T_{I J}^{*}(P, q) u_{J}(q) d \Gamma(q)- \\
\int_{\Omega_{l}-\Omega_{e}} \Delta C_{i J K l}(Q) \gamma_{K l}(Q) U_{I J, i}^{*}(P, Q) d \Omega(Q)- \\
\int_{\Omega_{e}} U_{I J, i}^{*}(P, Q)\left(\Delta C_{i J K l}(Q) \gamma_{K l}(Q)-\Delta C_{i J K l}(P) \gamma_{K l}(P)\right) d \Omega(Q)- \\
\int_{\Gamma_{e}} U_{I J}^{*}(P, q) n_{i}(q) \Delta C_{i J K l}(P) \gamma_{K l}(P) d \Gamma(q)
\end{gathered}
$$

The above integral equation may be safely differentiated with respect to the $\bar{l}$ coordinate of the source point $P$ since this operation gives rise to convergent integrals only [16]. One obtains

$$
\begin{gathered}
u_{I, \bar{l}}(P)=\int_{\Gamma} U_{I J, \bar{l}}^{*}(P, q) t_{J}(q) d \Gamma(q)-\int_{\Gamma} T_{I J, \bar{l}}^{*}(P, q) u_{J}(q) d \Gamma(q)- \\
\int_{\Omega_{l}-\Omega_{e}} \Delta C_{i J K l}(Q) \gamma_{K l}(Q) U_{I J, i \bar{l}}^{*}(P, Q) d \Omega(Q)- \\
\int_{\Omega_{e}} U_{I J, \bar{l}}^{*}(P, Q)\left(\Delta C_{i J K l}(Q) \gamma_{K l}(Q)-\Delta C_{i J K l}(P) \gamma_{K l}(P)\right) d \Omega(Q)- \\
\int_{\Gamma_{e}} U_{I J, \bar{l}}^{*}(P, q) n_{i}(q) \Delta C_{i J K l}(P) \gamma_{K l}(P) d \Gamma(q)
\end{gathered}
$$

Therefore, the extended strain tensor can be obtained 


$$
\begin{aligned}
& \gamma_{I j}(P)=\int_{\Gamma} U_{I j J}^{*}(P, q) t_{J}(q) d \Gamma(q)-\int_{\Gamma} T_{I j J}^{*}(P, q) u_{J}(q) d \Gamma(q)- \\
& \int_{\Omega_{l}-\Omega_{e}} \Delta C_{m J K l}(Q) \gamma_{K l}(Q) U_{I j m J}^{*}(P, Q) d \Omega(Q)- \\
& \int_{\Omega_{e}} U_{I j m J}^{*}(P, Q)\left(\Delta C_{m J K l}(Q) \gamma_{K l}(Q)-\Delta C_{m J K l}(P) \gamma_{K l}(P)\right) d \Omega(Q)- \\
& \int_{\Gamma_{e}} U_{I j J}^{*}(P, q) n_{m}(q) \Delta C_{m J K l}(P) \gamma_{K l}(P) d \Gamma(q)
\end{aligned}
$$

where

$$
\begin{gathered}
U_{I j J}^{*}=\left\{\begin{array}{cc}
\frac{1}{2}\left(U_{i J, \bar{j}}^{*}+U_{j J, \bar{i}}^{*}\right) & I=1,2,3 \\
U_{I J, \bar{j}}^{*} & I=4,5
\end{array}\right. \\
T_{I j J}^{*}=\left\{\begin{array}{cc}
\frac{1}{2}\left(T_{i J, \bar{j}}^{*}+T_{j J, \bar{i}}^{*}\right) & I=1,2,3 \\
T_{I J, \bar{j}}^{*} & I=4,5
\end{array}\right. \\
U_{I j m, J}^{*}=\left\{\begin{array}{cc}
\frac{1}{2}\left(U_{i J, m \bar{j}}^{*}+U_{j J, m \bar{i}}^{*}\right) & I=1,2,3 \\
U_{I J, m \bar{j}}^{*} & I=4,5
\end{array}\right.
\end{gathered}
$$

In eqn (20), $U_{I J, \bar{j}}^{*}$ and $T_{I J, \bar{j}}^{*}$ can be found in reference [1]. $U_{I J, m \bar{j}}^{*}$ has the following expression

$$
U_{I J, m \bar{j}}^{*}=-\frac{1}{\pi} \operatorname{Re}\left[A_{J M} H_{M I} \frac{\left(\delta_{1 i}+\mu_{M} \delta_{2 i}\right)\left(\delta_{1 \bar{l}}+\mu_{M} \delta_{2 \bar{l}}\right)}{\left(z_{M}^{q}-z_{M}^{P}\right)^{2}}\right]
$$

where $\boldsymbol{A}, \boldsymbol{H}$ and $\mu_{M}$ may be calculated by the method [17], and

$$
z_{M}^{q}=x_{1}(q)+\mu_{M} x_{2}(q), z_{M}^{P}=x_{1}(P)+\mu_{M} x_{2}(P)
$$

After discretizing eqns (14) and (19) using quadratic approximation for the generalized displacements, tractions along the matrix boundary and the generalized shear strains in inclusion, and all the unknowns are taken to the left hand side, the system of equations can be obtained

$$
\boldsymbol{A X}=\boldsymbol{F}
$$

where $\boldsymbol{A}$ is the matrix of coefficients; $\boldsymbol{X}$ is the vector of boundary unknowns and the extended strains in the inclusion; $\boldsymbol{F}$ is the known vector calculated by the product of the known boundary conditions and the corresponding matrix coefficients. 
After the unknowns are obtained using eqn (21), the extended stresses at points in the inclusion can be easily computed by eqn (1). For the points in the matrix, the corresponding extended stresses can be calculated by the following equation

$$
\sigma_{i J}=C_{i J K l}^{M} \gamma_{K l}
$$

where $\gamma_{K l}$ is calculated by

$$
\begin{aligned}
\gamma_{K l}(P)= & \int_{\Gamma} U_{K I J}^{*}(P, q) t_{J}(q) d \Gamma(q)-\int_{\Gamma} T_{K I J}^{*}(P, q) u_{J}(q) d \Gamma(q)- \\
& \int_{\Omega_{I}} \Delta C_{m J M n}(Q) \gamma_{M n}(Q) U_{K l m J}^{*}(P, Q) d \Omega(Q)
\end{aligned}
$$

Note that for infinite domain, eqn (19) can be simplified as

$$
\begin{aligned}
\gamma_{l j}(P)= & \gamma_{l j}^{0}(P)- \\
& \int_{\Omega_{e}} U_{I j m J}^{*}(P, Q)\left(\Delta C_{m J K l}(Q) \gamma_{K l}(Q)-\Delta C_{m J K l}(P) \gamma_{K l}(P)\right) d \Omega(Q)- \\
& \int_{\Gamma_{e}} U_{I j J}^{*}(P, q) n_{m}(q) \Delta C_{m J K l}(P) \gamma_{K l}(P) d \Gamma(q)
\end{aligned}
$$

where $\gamma_{K l}^{0}$ are the extended strain components by the remote extended loading.

For the more complicated inclusion-crack medium, the above related equations can be expanded to study the interaction between the inclusions and cracks, i.e. for point on the matrix outer boundary

$$
\begin{aligned}
C_{I J}(P) u_{J}(P)= & \int_{\Gamma} U_{I J}^{*}(P, q) t_{J}(q) d \Gamma(q)-\int_{\Gamma} T_{I J}^{*}(P, q) u_{J}(q) d \Gamma(q)- \\
& \int_{\Gamma_{c}} T_{I J}^{*}(P, q) \Delta u_{J}(q) d \Gamma(q)-\int_{\Omega_{I}} \Delta C_{i J K l}(Q) \gamma_{K l}(Q) U_{I J, i}^{*}(P, Q) d \Omega(Q)
\end{aligned}
$$

where the extended discontinuous displacements $\Delta u_{J}$ over the crack $\Gamma_{c}$ are as follows

$$
\Delta u_{J}=\left\{\begin{array}{l}
u_{1}^{+}-u_{1}^{-} \\
u_{2}^{+}-u_{2}^{-} \\
u_{3}^{+}-u_{3}^{-} \\
\varphi^{+}-\varphi^{-} \\
\phi^{+}-\phi^{-}
\end{array}\right\}
$$

where the superscripts + and - stand for the upper and lower crack surfaces, respectively. $u_{i}, \varphi$ and $\phi$ are respectively the elastic displacement, the electric 
potential and the magnetic potential. Note that $t_{J}^{+}+t_{J}^{-}=0$ has been assumed in the derivation of eqn (25).

For point in the inclusion, one has

$$
\begin{aligned}
& \gamma_{I j}(P)=\int_{\Gamma} U_{l j J}^{*}(P, q) t_{J}(q) d \Gamma(q)-\int_{\Gamma} T_{I j J}^{*}(P, q) u_{J}(q) d \Gamma(q)- \\
& \quad \int_{\Gamma_{c}} T_{I i J}^{*}(P, q) \Delta u_{J}(q) d \Gamma(q)-\int_{\Omega_{l}-\Omega_{e}} \Delta C_{m J K l}(Q) \gamma_{K l}(Q) U_{I j m J}^{*}(P, Q) d \Omega(Q)-(27) \\
& \int_{\Omega_{e}} U_{I j m J}^{*}(P, Q)\left(\Delta C_{m J K l}(Q) \gamma_{K l}(Q)-\Delta C_{m J K l}(P) \gamma_{K l}(P)\right) d \Omega(Q)- \\
& \int_{\Gamma_{e}} U_{l j J}^{*}(P, q) n_{m}(q) \Delta C_{m J K l}(P) \gamma_{K l}(P) d \Gamma(q)
\end{aligned}
$$

For point over the crack, one has

$$
\begin{aligned}
\sigma_{i J}(P)= & \int_{\Gamma} C_{i J J j}^{M} U_{l j N}^{*}(P, q) t_{N}(q) d \Gamma(q)-\int_{\Gamma} C_{i J J j}^{M} T_{I i N}^{*}(P, q) u_{N}(q) d \Gamma(q)- \\
& \int_{\Gamma_{c}} C_{i J J j}^{M} T_{I i N}^{*}(P, q) \Delta u_{N}(q) d \Gamma(q)- \\
& \int_{\Omega_{I}} C_{i J j}^{M} \Delta C_{m N K l}(Q) \gamma_{K l}(Q) U_{I j m N}^{*}(P, Q) d \Omega(Q)
\end{aligned}
$$

where the third integral in the right hand side of eqn (28) contains the hypersingular integral which can be calculated by the existing method [1].

Eqns (25), (27) and (28) can be used to investigate the interaction between the inclusions and cracks. For infinite domain, only the simplified eqns (27) and (28) need to be adopted to study inhomogeneous media, i.e.

$$
\begin{gathered}
\gamma_{l j}(P)=\gamma_{l j}^{0}(P)-\int_{\Gamma_{c}} T_{I j J}^{*}(P, q) \Delta u_{J}(q) d \Gamma(q)- \\
\int_{\Omega_{l}-\Omega_{e}} \Delta C_{m J K l}(Q) \gamma_{K l}(Q) U_{I j m J}^{*}(P, Q) d \Omega(Q)- \\
\int_{\Omega_{e}} U_{I j m J}^{*}(P, Q)\left(\Delta C_{m J K l}(Q) \gamma_{K l}(Q)-\Delta C_{m K K l}(P) \gamma_{K l}(P)\right) d \Omega(Q)- \\
\int_{\Gamma_{e}} U_{I j J}^{*}(P, q) n_{m}(q) \Delta C_{m J K l}(P) \gamma_{K l}(P) d \Gamma(q) \\
\sigma_{i J}(P)=\sigma_{i J}^{0}(P)-\int_{\Gamma_{c}} C_{i J J j}^{M} T_{I j N}^{*}(P, q) \Delta u_{N}(q) d \Gamma(q)- \\
\quad \int_{\Omega_{l}} C_{i J J j}^{M} \Delta C_{m N K l}(Q) \gamma_{K l}(Q) U_{I j m N}^{*}(P, Q) d \Omega(Q)
\end{gathered}
$$

where $\sigma_{i J}^{0}$ are the extended stress components by the remote extended loading.

Once the extended discontinuous displacement components over crack are obtained, the extended stress intensity factors can be easily calculated by using the existing formulations [1]. 


\section{Numerical example}

A circular inclusion and a horizontal crack along $\mathrm{x}$ axis are embedded into an infinite magneto-electro-elastic matrix under a uniform far-field stress or an electric displacement or a magnetic induction (see Figure 1). The material parameters from the matrix and inclusion are given as shown in Tables 1 and 3 (see [12]), respectively. The inclusion with radius $\mathrm{a}=1 \mathrm{~m}$ is meshed into 8 quadratic quadrilateral elements and 8 quadratic triangular elements. The crack surface is discretized into 20 discontinuous quadratic elements. Under the uniform remote stress or the electric displacement or the magnetic induction, the stress $\sigma_{y y}$, electric displacement $D_{y}$ and magnetic induction $B_{y}$ within the inclusion, the normal discontinuous displacement $\Delta u_{n}$, the discontinuous electric potential $\Delta \varphi$ and the discontinuous magnetic potential $\Delta \phi$ over the crack are respectively shown in Figures $2-7$. Interaction between the inclusion and crack can be easily observed.

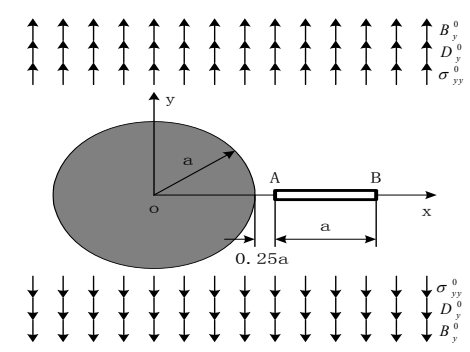

Figure 1: A circular inclusion and a straight crack.

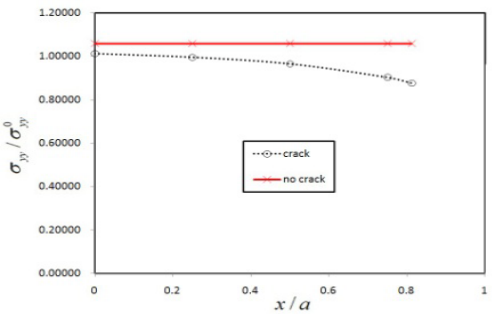

Figure 2: $\quad$ Stress $\sigma_{y y}$ along $\mathrm{x}$-axis in inclusion under remote stress $\sigma_{y y}^{0}$.

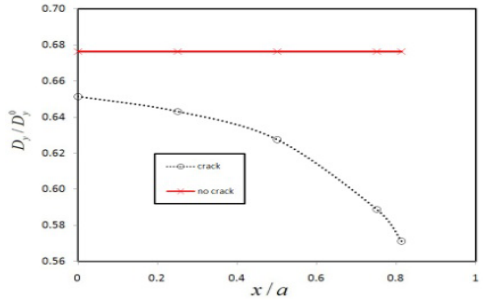

Figure 3: Electric displacement $D_{y}$ along $\mathrm{x}$-axis in inclusion under remote electric displacement $D_{y}^{0}$. 


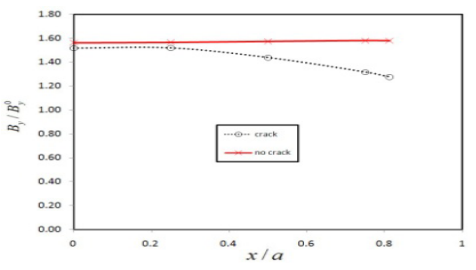

Figure 4: Magnetic induction $B_{y}$ along $\mathrm{x}$-axis in inclusion under remote magnetic induction $B_{y}^{0}$.

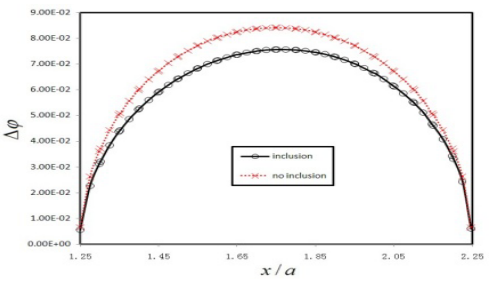

Figure 6: Discontinuous electric potential $\Delta \varphi$ along crack under remote Electric displacement $D_{y}^{0}$.

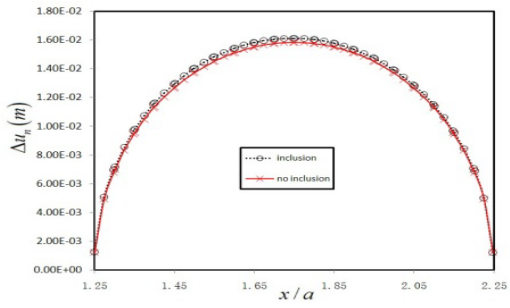

Figure 5: Normal discontinuous displacement $\Delta u_{n}$ along crack under remote stress $\sigma_{y y}^{0}$.

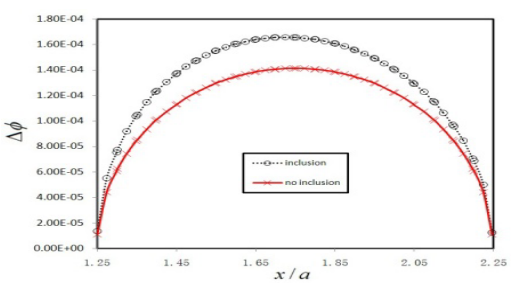

Figure 7: Discontinuous electric potential $\Delta \phi \quad$ along crack under remote magnetic induction $B_{y}^{0}$.

\section{Conclusions}

Integral equation formulations for 2D inhomogeneous finite/infinite magnetoelectroelastic media have been presented. The present formulations can be used to investigate the interaction between the inclusions and cracks, and can be also used to carry out the analysis of effective properties of magnetoelectroelastic media. The inclusions may be exponentially (but not limited to) functionally graded magnetoelectroelastic material. The present formulations only need the fundamental solutions from the homogeneous anisotropic magnetoelectroelastic matrix which are available in the literature. More examples will be reported later.

\section{Acknowledgement}

The project is supported by the National Natural Science Foundation of China (11072034, 11132011, 11272054). 


\section{References}

[1] Garcia-Sanchez, F., Rojas-Diaz, R., Saez, A. and Zhang, Ch., Fracture of magnetoelectroelastic composite materials using boundary element method (BEM). Theoretical and Applied Fracture Mechanics, 47, pp. 192-204, 2007.

[2] Ding, H.J. and Jiang, A.M., A boundary integral formulation and solution for 2D problems in magneto-electro-elastic media. Computers and Structures, 82, pp. 1599-1607, 2004.

[3] Huang, J.H. and Kuo, W.S., The analysis of piezoelectric/piezomagnetic composite materials containing ellipsoidal inclusions. Journal of Applied Physics, 81, pp. 1378-1386, 1997.

[4] Huang, J.H., Chiu, Y.H. and Liu, H.K., Magneto-electro-elastic Eshelby tensors for a piezoelectric-piezomagnetic composite reinforced by ellipsoid inclusions. Journal of Applied Physics, 83, pp. 5364-5370, 1998.

[5] Li, J.Y., Magneto-electro-elastic multi-inclusion and inhomogeneity problems and their applications in composite materials. International Journal of Engineering Science, 38, pp. 1993-2011, 2000.

[6] Liu, J.X., Liu, X.L. and Zhao, Y.B., Green's functions for anisotropic magnetoelectroelastic solids with an elliptical cavity or a crack. International Journal of Engineering Science, 39, pp. 1405-1418, 2001.

[7] Pan, E., Three-dimensional Green's functions in anisotropic magnetoelectro-elastic bimaterials. Journal of Applied Mathematics and Physics (ZAMP), 53, pp. 815-838, 2002.

[8] Jiang, X. and Pan, E., Exact solution for 2D polygonal inclusion problem in anisotropic magnetoelectroelastic full-, half-, and bimaterial-planes. International Journal of Solids and Structures, 41, pp. 4361-4382, 2004.

[9] Hou, P.F. and Leung, A.Y.T., A spheroidal inclusion in an infinite magneto-electro-elastic material. International Journal of Engineering Science, 42, pp. 1255-1273, 2004.

[10] Dinzart, F. and Sabar, H., Magneto-electro-elastic coated inclusion problem and its application to magnetic-piezoelectric composite materials. International Journal of Solids and Structures, 48, pp. 2393-2401, 2011.

[11] Shen, M.H. and Hung, S.Y., Magnetoelectroelastic analysis of an arbitrary shape inclusion undergoing eigenfields and remote loadings. International Journal of Solids and Structures, 49, pp. 3808-3815, 2012.

[12] Dong, C.Y., Lo, S.H. and Antes, H., Fracture analysis in 2D magnetoelectro-elastic media by the boundary element method. Computational Mechanics, 41, pp. 207-217, 2008.

[13] Pasternak, I., Doubly periodic arrays of cracks and thin inhomogeneities in an infinite magnetoelectroelastic medium. Engineering Analysis with Boundary Elements, 36, pp. 799-811, 2012.

[14] Rojas-Diaz, R., Denda, M., Garcia-Sanchez, F. and Saez, A., Dual BEM analysis of different crack face boundary conditions in 2D magnetoelectroelastic solids. European Journal of Mechanics A/Solids, 31, pp. 152-162, 2012. 
[15] Brebbia, C.A. and Dominguez, J., Boundary Elements - An Introduction Course. Computational Mechanics Publications: Southampton, 1992.

[16] Dong, C. and Bonnet, M., An integral formulation for steady-state elastoplastic contact over a coated half-plane. Computational Mechanics, 28, pp. 105-121, 2002.

[17] Barnett, D.M. and Lothe, J., Synthesis of the sextic and the integral formalism for dislocations, Green functions, and surface waves in anisotropic elastic solids. Physica Norvegica, 7, pp. 13-19, 1973. 